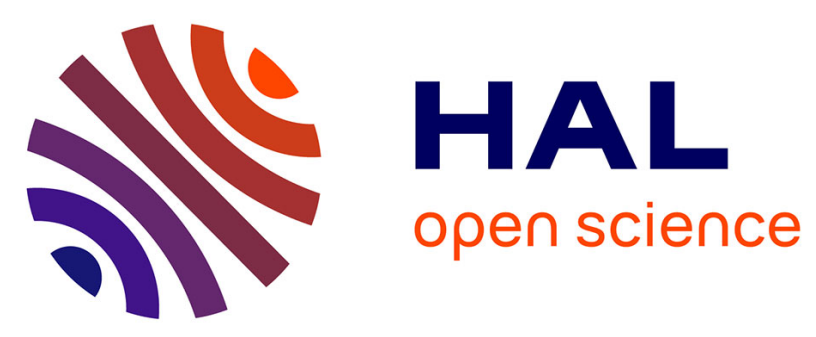

\title{
Mannose-coated Fluorescent Lipid Microparticles for Specific Cellular Targeting and Internalization via Glycoreceptor-Induced Phagocytosis
}

Blaise Dumat, Lorraine Montel, Léa Pinon, Pascal Matton, Laurent Cattiaux, Jacques Fattaccioli, Jean-Maurice Mallet

\section{To cite this version:}

Blaise Dumat, Lorraine Montel, Léa Pinon, Pascal Matton, Laurent Cattiaux, et al.. Mannose-coated Fluorescent Lipid Microparticles for Specific Cellular Targeting and Internalization via GlycoreceptorInduced Phagocytosis. ACS Applied Bio Materials, 2019, 10.1021/acsabm.9b00793 . hal-02338500

\section{HAL Id: hal-02338500 https://hal.science/hal-02338500}

Submitted on 30 Nov 2020

HAL is a multi-disciplinary open access archive for the deposit and dissemination of scientific research documents, whether they are published or not. The documents may come from teaching and research institutions in France or abroad, or from public or private research centers.
L'archive ouverte pluridisciplinaire HAL, est destinée au dépôt et à la diffusion de documents scientifiques de niveau recherche, publiés ou non, émanant des établissements d'enseignement et de recherche français ou étrangers, des laboratoires publics ou privés. 


\section{Mannose-coated Fluorescent Lipid Microparticles for Specific Cellular}

Targeting and Internalization via Glycoreceptor-Induced Phagocytosis

Blaise Dumat ${ }^{\dagger, \#}$, Lorraine Montel ${ }^{\ddagger, \S, \#, ~ L e ́ a ~ P i n o n ~}{ }^{\ddagger, \S}$, Pascal Matton $^{\dagger}$, Laurent Cattiaux ${ }^{\dagger}$, Jacques Fattaccioli ${ }^{\ddagger, \S,}$, Jean-Maurice Mallet ${ }^{\dagger,}$

† Laboratoire des Biomolécules, LBM, Département de Chimie, École Normale Supérieure, PSL University, Sorbonne Université, CNRS, 75005 Paris, France

‡ PASTEUR, Département de Chimie, École Normale Supérieure, PSL University, Sorbonne Université, CNRS, 75005 Paris, France

$\S$ Institut Pierre-Gilles de Gennes pour la Microfluidique, 75005 Paris, France

\# these authors contributed equally to this work

Corresponding authors : jacques.fattaccioli@ens.fr, jean-maurice.mallet@ens.fr 


\begin{abstract}
In this work we report on the development of mannose-coated fluorescent lipid microparticles to study the role of C-type lectin membrane receptor in phagocytosis. The micrometric droplets of soybean oil in water emulsion were functionalized with a tailor-made fluorescent mannolipid. The amphiphilic ligand was built from a mannose unit, a lipid C11 spacer and a naphthalimide fluorophore. The droplets functionalization was monitored by fluorescence microscopy as well as the interaction with concanavalin A which was used as a model lectin in vitro. The use of a monovalent ligand on the surface of emulsion droplets yielded particles with an affinity approximately 40 times higher than that of free mannose. In cellulo, the coated droplets were shown to be specifically internalized by macrophages in a receptor-dependent phagocytic pathway. The naked droplets on the other hand display very little internalization due to their low immunogenicity. This work thus brings evidence that C-type lectin membrane receptors may act as phagocytic receptors. The droplets functionalization with the tailored amphiphilic fluorescent ligand and the droplets functionalization also provides insights into the development of organic fluorescent particles that may prove useful for developing targeted imaging and delivery tools.
\end{abstract}

Keywords: oil-in-water emulsion droplets; fluorescent glycolipids; phagocytosis; carbohydrateslectins interactions; mannose receptor 


\section{Introduction}

Phagocytosis is the process by which cells internalize objects larger than 0.5 microns. Whereas unicellular organisms use phagocytosis to capture and eat preys, in multicellular organisms it represents a fundamental part of innate immunity and organ homeostasis. Innate immunity relies on a specialized subset of cells, the phagocytes, which patrol the organism, identify, ingest and eliminate pathogens. Among them, macrophages are versatile cells residing in tissues that are able to scavenge worn-out cells and participate to the activation of the adaptive immune response. Phagocytosis by macrophages is triggered by the binding of the target to specific receptors present at the surface of the phagocyte. Several receptors have been identified so far, each involving different signaling pathways and ingestion mechanisms. Opsonic receptors rely on the recognition of the pathogen by immunoglobulin or complement proteins. In the case of the Fcy receptorsmediated phagocytosis, antigens present at the surface of the target are bound by specific soluble immunoglobulins (IgGs). ${ }^{1} \mathrm{Fc}$ regions of those $\mathrm{IgGs}$ are then actively recognized by the Fcy receptors at the surface of the phagocyte, which form clusters and trigger the internalization. ${ }^{2-4}$ Engulfment then occurs by an actin-driven membrane extension and closure of a phagocytic cup around the foreign body to create a specific degradative compartment: the phagosome..$^{5,6}$

While FcyR-mediated phagocytosis relies on specific recognition by $\lg G$ during the secondary immune response, Pattern Recognition Receptors recognize conserved pathogen-associated molecular patterns (PAMPs) on the surface of bacteria and yeasts to ensure innate immunity. ${ }^{7}$ Carbohydrate receptors like the Mannose Receptor (MR) family or DC-SIGN are C-type lectins that bind glycoconjugates present in cell walls in a $\mathrm{Ca}^{2+}$ dependent manner.

MR is present in macrophages and targets polysaccharide patterns with terminal mannose, fucose and $\mathrm{N}$-Acetylglucosamine (GlcNAc). ${ }^{8} \mathrm{DC}$-SIGN is a receptor primarily expressed in dendritic cells but also in some sub-populations of macrophages that targets terminal mannose groups like mannans. ${ }^{7,9}$ Their role in phagocytosis, and especially their ability to trigger it, is unclear as of yet. They have been identified in phagosomes ${ }^{10,11}$ and shown to participate in the binding and internalization of targets. ${ }^{12-14}$ However, neither could induce phagocytosis in non-phagocytic cell lines, and whether they can trigger phagocytosis on their own or merely cooperate with other receptors to do so remains an open question. ${ }^{15}$

Macrophage activity can be detected by small molecular fluorescent probes reporting on $\mathrm{pH}$ acidification or ROS production inside the phagosome. ${ }^{16-19}$ Such probes however only report indirectly on processes posterior to the internalization. The adhesion and internalization steps of phagocytosis involve large three dimensional biological objects (cells, apoptotic bodies, bacteria) 
and can only be probed directly by large micrometric particulate biosensors. Nanoparticles and nanostructures have become platforms of choice to build up biosensors or delivery systems incorporating several probes, ligands, or cargo within the same object. ${ }^{20-23}$ Although less used, microparticles can be made in similar fashion and come in solid (e.g. metallic particles, silica or polystyrene beads) or liquid form (e.g. liposomes, emulsions). Liquid particles have the distinct advantage of exhibiting a fluid interface similar to that of cellular lipid bilayers and they can reproduce the mobility of interfacial ligands and receptors during phagocytosis. To that end, liposomes are by far the most used liquid particles in biology, with numerous examples of cell receptor recognition by glycosylated structures. ${ }^{24-26}$ Alternatively, oil-in-water (ON) emulsions made out of vegetable oil are easily accessible and biocompatible particles. They have been used since half a century as colloidal drug carriers for various therapeutic applications and they can be fabricated with a narrow size distribution ranging from a few tenth of nanometers to several hundreds of microns. ${ }^{27,28}$ Carefully functionalized with biologically-relevant adhesive molecules, they are able to specifically interact with cells ${ }^{29}$ and can be used as model particles for cell adhesion modeling. ${ }^{30-32}$ These properties make them good candidates to build up targeted platforms for imaging or therapeutic applications. Yet, turning emulsion droplets into biosensors requires controlled chemical surface functionalization with appropriate reporters and targeting moieties and such examples are still scarce. ${ }^{33}$

We report herein on the development of fluorescent lipid particles targeted towards receptors from the lectin family based on micrometric $\mathrm{O} / \mathrm{W}$ emulsion droplets functionalized with a tailor-made glycosylated fluorescent ligand. We have synthesized a fluorescent mannolipid consisting of a naphthalimide fluorophore, a C-11 spacer and a mannose and, after careful characterization of its optical properties, the glycolipid was used to functionalize the surface of micrometric oil-in-water emulsion droplets. We show that the resulting particles interact selectively in vitro with a model lectin protein and that they are specifically internalized by macrophages via glycoreceptor-mediated phagocytosis.

\section{RESULTS AND DISCUSSION}

Design and synthesis of the fluorescence mannolipid. Lectins are a promising class of biomarkers (for bacteria or cancer cells detection for instance) and a number of synthetic ligands have been reported, ${ }^{34}$ some of them being fluorescent for targeted imaging of cell receptors. ${ }^{35,36}$ Lectins however bind high-order glycan structures (such as mannans for DC-SIGN) while the affinity for monosaccharide units is low. ${ }^{37}$ The development of synthetic ligands targeting glycoreceptors with high affinity is thus a very demanding and time-consuming endeavor to produce dendrimers or oligomers. ${ }^{38,39}$ The aim of this project is to take advantage of the ligand mobility at the surface of the droplet, which can lead to the clustering of multiple ligands upon interaction with a multivalent 
receptor. It has been shown for instance on mannosylated liposomes that divalent ligands were as efficient for the recognition of dendritic cells than tetravalent ligands and that above a certain surface concentrations, monovalent ligand also yield good interactions. ${ }^{26}$ To take advantage of the clustering effect, we have thus developed a monovalent mannolipid which is simpler and more costeffective to synthesize and is expected in a supramolecular context to exhibit a high affinity for the target receptors.

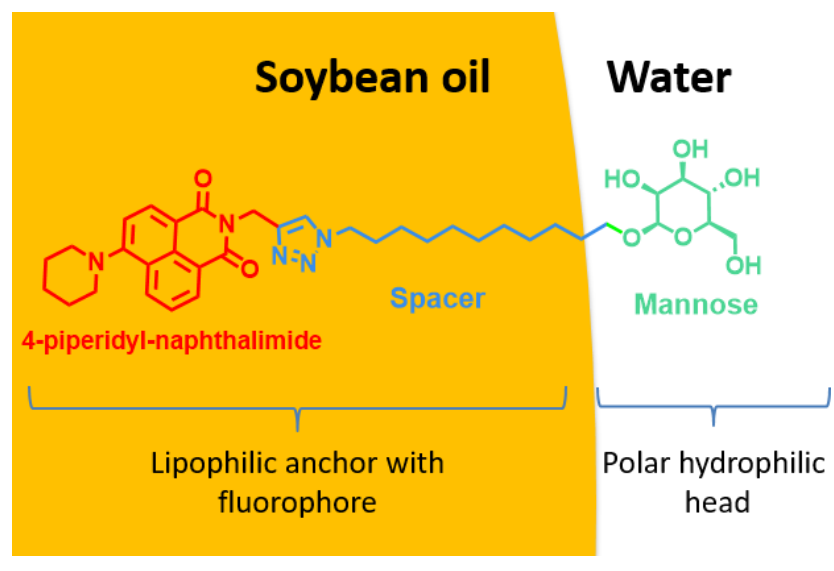

Figure 1. Structure of the mannolipid and expected positioning at the oil/water interface. A fluorescent dye is attached to the lipophilic anchor.

To efficiently coat the surface of the droplets, the sugar moiety had to be tethered to a lipophilic anchor (Figure 1). Moreover, in order to characterize the functionalization of the droplets as well as to monitor their cellular fate using fluorescence microscopy, we chose to incorporate a fluorophore moiety. The mannolipid 1 was thus built from three parts: 11-azido undecanol as a lipophilic spacer, sugar block 6 and alkyne-functionalized piperidyl naphthalimide fluorophore 4 (Scheme 1). The naphthalimide fluorescent scaffold was selected for its photostability and ease of functionalization: 4-bromo-1,8-naphthalic anhydride commercial derivative can be easily and selectively derivatized via the bromine and anhydride groups. Moreover, naphthalimide derivatives have been extensively characterized and their photophysical properties are well understood. ${ }^{40,41}$ The introduction of an amino group at the $\mathbf{4}$ position creates a push-pull structures and endows the naphthalimide with a solvatochromic and environment-sensitive fluorescence emission particularly suited for biological imaging. A major advantage of push-pull probes is their low fluorescence in water and polar solvent which make them particularly apt to probe hydrophobic environments or in our case to track $\mathrm{O} / \mathrm{W}$ emulsion droplets. The choice of a cyclic amine substituent in position 4 is aimed at inhibiting twisted intramolecular charge transfer (TICT) commonly observed with dialkylamino electron donating group. Inhibition of TICT results in higher brightness and superior photostability. ${ }^{41}$ Piperidine was chosen since more strained cyclic amines with three or four carbon rings result in high fluorescence quantum yields in water which is not a desired property in this work. 


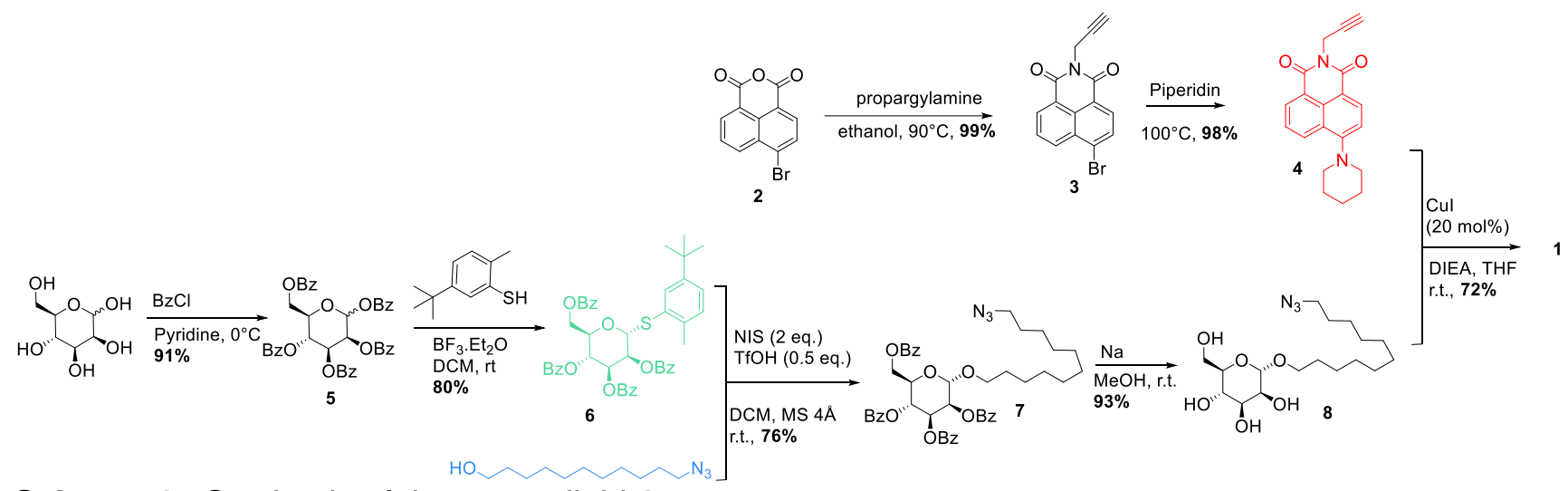

Scheme 1: Synthesis of the mannolipid 1

Glycoside 7 was synthesized using glycosylation of 11 -azido-undecanol with donor 6 using the odorless 2-methyl-5 terbutyl thiophenol strategy. The presence of the benzoate group in position 2 which participates in the glycosylation mechanism yields the $\alpha$ anomer (Carbon NMR: $\delta\left(C_{\text {anomeric }}\right)=$ 99.7 ppm). Compound 7 was deprotected using sodium methoxide to give $\mathbf{8}$ which then reacted with 7 in a copper-catalyzed azide-alkyne cycloaddition to afford the mannolipid 1 (scheme 1). This highyielding convergent synthesis using widely applicable chemical functionalities (alcohol and azide/alkyne) offers the possibility to independently change any of the three components (sugar, spacer, fluorophore) of the molecule in future developments.

Charaterization of the fluorescent mannolipid. We first characterized the optical properties of the fluorescent mannolipid in water, in ethanol and in the soybean oil used for the fabrication of the emulsion droplets (Figure 2). Intermediate 4 was also studied in ethanol and in soybean oil (figure S1). The results are summarized in table 1. Naphthalimide 4 displays an intense and broad absorption band with a positive solvatochromism characteristic of an intramolecular charge transfer (ICT) band, due to the dipolar structure of the chromophore. In ethanol it emits a weak fluorescence signal centered on $545 \mathrm{~nm}$, with a very large stokes shift. In soybean oil, the fluorescence signal is blue-shifted and much brighter with a structured emission spectrum showing a maximum at $495 \mathrm{~nm}$ and a shoulder peak around $530 \mathrm{~nm}$. Both emission peaks have similar excitation spectra and thus result from an excited-state phenomenon. Dipolar chromophore usually emit from a relaxed excited state resulting from stabilizing interactions of the solvent molecules with the excited state which displays a stronger dipolar moment than the ground state. We assume that, due to the oil viscosity, the relaxation rate is in the same range as the radiative deactivation rate and thus the relaxed excited state coexists with the blue-shifted locally excited (LE) state giving two emission peaks.

Table 1 : Spectroscopic data of 1 and 4 in various solvents. $\lambda_{a b s:}$ peak absorption wavelength, $\varepsilon$ : molar absorption coefficient, $\lambda_{\text {em }}$ : peak emission wavelength (sh: shoulder peak), $\Delta \lambda$ : stokes shift in $\mathrm{nm}, \Phi_{\mathrm{F}}$ : fluorescence quantum yield.

\begin{tabular}{|c|c|c|c|c|c|c|c|}
\hline Solvent & Molecule & $\lambda_{\text {abs }}$ & $\varepsilon$ & $\lambda_{\mathrm{em}}$ & $\Delta \lambda$ & $\Phi_{\mathrm{F}}$ & Brightness \\
\hline
\end{tabular}




\begin{tabular}{|c|l|l|l|l|l|l|c|}
\hline & & $(\mathrm{nm})$ & $\left(\mathrm{M}^{-1} \cdot \mathrm{cm}^{-1}\right)$ & \multicolumn{1}{|c|}{$(\mathrm{nm})$} & \multicolumn{1}{|c|}{$(\mathrm{nm})$} & & $\left(\varepsilon \cdot \Phi_{\mathrm{F}}\right)$ \\
\hline \multirow{2}{*}{$\mathrm{EtOH}$} & $\mathbf{1}$ & 410 & 11100 & 545 & 135 & 0.016 & 180 \\
\cline { 2 - 8 } & $\mathbf{4}$ & 411 & 10100 & 545 & 134 & 0.014 & 140 \\
\hline \multirow{2}{*}{ Soybean oil } & $\mathbf{1}$ & 402 & 3500 & $495(\mathrm{sh} 530)$ & $93(128)$ & 0.71 & 2500 \\
\cline { 2 - 8 } & $\mathbf{4}$ & 400 & 11000 & $495(\mathrm{sh} 530)$ & $95(130)$ & 0.72 & 7900 \\
\hline Water & $\mathbf{1}$ & 419 & 3300 & 553 & 134 & $(0.070)^{\mathrm{a}}$ & 230 \\
\hline
\end{tabular}

(a) In water, the $\mathrm{cmc}$ is too low and there is a poor linearity between the fluorescence and absorption of 1 ; the value of $\Phi_{\mathrm{F}}$ is thus a rough estimate given only for comparison purposes.

The optical properties of the mannolipid $\mathbf{1}$ in ethanol are essentially identical to those of intermediate 4. On the other hand, significant differences are observed in water or in soybean oil. Compared to intermediate 4 , the mannolipid displays a strong hypochromism in soybean oil but the quantum yield remains essentially identical as well as the structured emission spectrum (Figure 2). In water the absorptivity of $\mathbf{1}$ is similar to that in oil and although it cannot be compared to $\mathbf{4}$, it suggests a similar hypochromism. The fluorescence intensity does not vary linearly with the absorption and the estimated quantum yield in water is also quite high for such a push-pull probe in water (Figure S2). ${ }^{41}$ These properties can arise from solubility issues like aggregation or from the formation of self-assembled structures due to the amphiphilic character of the molecule.
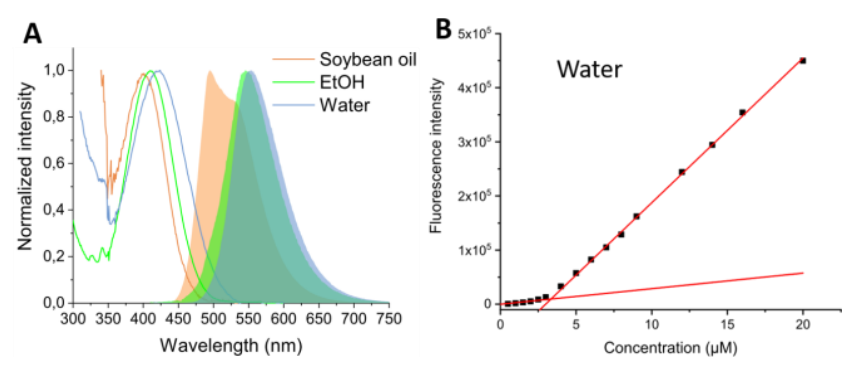

Figure 2. (A) Spectral properties of the mannolipid in ethanol, water and soybean oil. Normalized absorption and fluorescence emission spectra. (B) Fluorescence intensity as a function of concentration in water.

We have thus investigated the formation of micelles in water by fluorescence spectroscopy (see figure 2B). Two linear regimes were identified, corresponding to the solute and micellar states of the mannolipid with a $\mathrm{cmc}$ of $3.3 \mu \mathrm{M}$. The micellar state is more emissive, which explains the fact that the measured quantum yield is higher than expected. A similar experiment in soybean oil reveals no signs of self-assembly and the mannolipid is soluble in oil at least up to $50 \mu \mathrm{M}$ (Figure S3).

Overall, the fluorescent mannolipid displays a low fluorescence emission in polar solvents such as ethanol and water and a high brightness in the soybean oil used for fabricating the droplets. It is poorly soluble in water and well soluble in oil up to $50 \mu \mathrm{M}$ at least. These results show that 
compound 1 is well-suited for functionalizing O/W emulsion droplets with high contrast: should an excess of mannolipid remain in the bulk phase, it is expected to yield a low fluorescence signal. We next studied the functionalization of emulsion droplets by the mannolipid in order to create multivalent mannosylated fluorescent microparticles able to specifically target mannose receptors such as MR or DC-SIGN at the cell surface.

Functionalization of droplets. To characterize the ability of the mannolipid to functionalize the $\mathrm{O} / \mathrm{W}$ emulsion, we studied its insertion on the surface of micrometric oil droplets of $10 \mu \mathrm{m}$ diameter by fluorescence microscopy. The droplets were functionalized by the amphiphilic ligand using a recently reported procedure that allows rapid and efficient coating of $\mathrm{O} / \mathrm{W}$ emulsion. ${ }^{42}$ While most surface coating protocols rely on the in-situ fabrication of the emulsion with the functional ligand, our protocol allows functionalizing the droplets after the emulsification. Briefly, the droplets can be fabricated in a Couette emulsifier with the desired size ${ }^{43}$ and then functionalized by being put in the presence of the ligand in a polar cosolvent (here $4 \% \mathrm{v} / \mathrm{v}$ DMSO) during 30 minutes. $^{42}$ It is thus possible to rapidly functionalize the droplets at various concentrations in mannolipid and to perform a thorough study of the surface coating with a single batch of droplets.
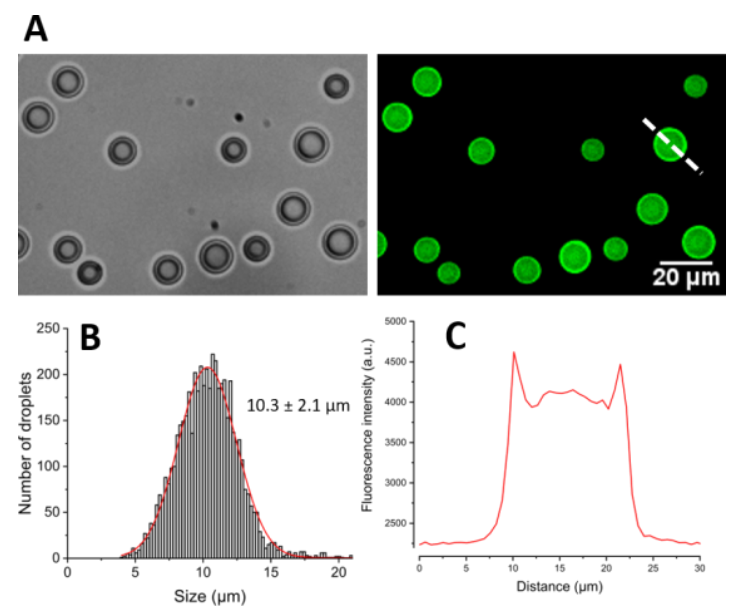

Figure 3. (A) Transmission, epifluorescence and overlay microscopy images (40X objective) of $10 \mu \mathrm{m}$ emulsion droplets functionalized with 1 surface equivalent of mannolipid. (B) Size distribution of the droplets measured using fluorescence images and Gaussian fit (red curve). (C) Plot of the fluorescence intensity profile along the dashed lines shown in $(A)$.

The droplets were put in presence of increasing concentrations of mannolipid from 20 to $400 \mu \mathrm{M}$ in the buffer. Since molar concentrations are a poor description for surface functionalization, we have converted the concentrations into surface equivalents assuming a surface excess of $50 \AA^{2}$ for the mannolipid as was previously reported for linear glycolipid with a single sugar unit and comparable chain lengths. ${ }^{44}$ We worked with $2.3 \times 10^{7}$ droplets of $10 \mu \mathrm{m}$ diameter, which corresponds to $7.2 \times 10^{9}$ 
$\mu \mathrm{m}^{2}$ of total droplets surface. In these conditions, 1 surface equivalent corresponds to $100 \mu \mathrm{M}$ of mannolipid in a $250 \mu \mathrm{L}$ working volume.

Figure 3A shows pictures of droplets functionalized with 1 surface equivalent. The droplets display a fluorescence emission excited in the DAPI channel ( $387 \pm 11 \mathrm{~nm}$ BP excitation filter) evidencing the successful surface coating by the mannolipid. The fluorescence images were used to analyze the particles and measure the size distribution of the droplets (Figure 3B). A Gaussian fit yielded an average size of $10.3 \pm 2.1 \mu \mathrm{m}$. The droplets are thus fairly monodispersed. The plot of the intensity profile along the diameter of a droplet (Figure 3C) shows a slightly higher intensity on the edges of the droplets showing that the mannolipid indeed inserts on the surface as expected. The low contrast is expected from the wide-field collection of the emitted light.

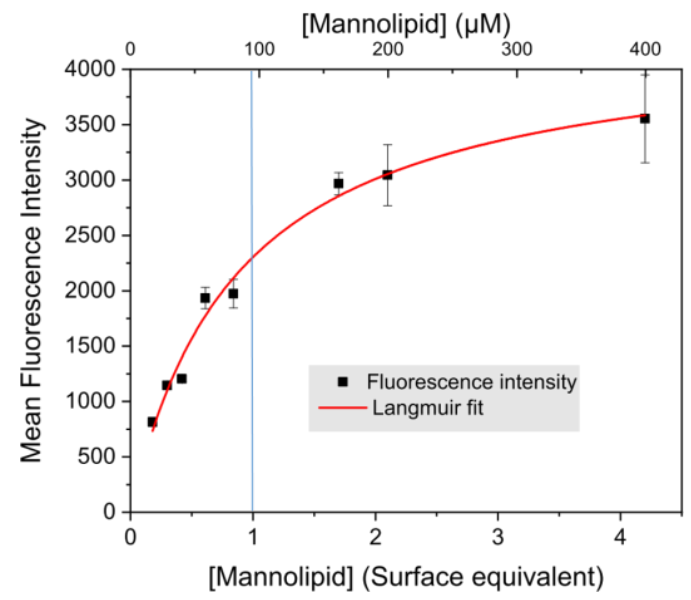

Figure 4: Titration of the mannolipid adsorption on the surface of $10 \mu \mathrm{m}$ droplets. Error bars represents the standard deviation to the mean intensity measured on 5 wide-field microscopy images for each datapoint. Red line is a fit of the titration curve to a Langmuir adsorption isotherm (see Supplementary Materials Table S1 for detailed nonlinear curve fitting results). The two x-axes give the mannolipid concentration in micromolar as well as the corresponding surface equivalents (see text for explanation).

A common issue when loading particles with fluorescent dyes is the self-quenching observed when the dyes are in close contact with one another. ${ }^{45}$ Here, interestingly, we do not observe any extinction of the fluorescence signal when increasing the amount of mannolipid on the particles even at the concentration where the surface is theoretically packed at maximum capacity and above. The mean fluorescence intensity plotted against the concentration of mannolipid follows a classical binding curve that can be fitted to a Langmuir isotherm, with a dissociation constant $K_{D}=$ $0.88 \pm 0.10$ surface eq. (Figure 4 ).

These results show that the emulsion droplets are efficiently coated by the fluorescent mannolipid without impacting its fluorescent properties, thus affording bright and stable fluorescent particles. The functionalized droplets can be stored at least for 24 hours in the fridge without apparent 
modification of their size and functionalization and they are photostable under epifluorescence illumination.

Interaction with concanavalin $\mathbf{A}$. The selective recognition of a mannose-specific receptor by the coated droplets was assessed in vitro with concanavalin A (ConA). ConA is a tetravalent lectin of the same sub-type (C-type lectins, active only upon calcium binding) as the Mannose Receptor or DC-SIGN. ${ }^{8}$ The Mannose Receptor, DC-SIGN and ConA bind $\alpha$-D-mannosyl (amongst other glycosylated structures) residues in terminal positions of large structures like glycoproteins or oligosaccharides. ConA can also interact with isolated D-mannose albeit with a much lower affinity, with reported $\mathrm{Kd}$ values of $360 \mu \mathrm{M}$ for $\alpha$-D-mannopyranoside or $131 \mu \mathrm{M}$ for methyl- $\alpha$-Dmannopyranoside. ${ }^{37,46}$ ConA was thus chosen as a model since it is an easily accessible and wellstudied lectin with similar properties as the target cellular membrane receptors.

Mannose-coated droplets were incubated with Alexa647-conjugated ConA ( $0.24 \mu \mathrm{M})$ for 30 minutes and, after washing the excess protein, observed under the microscope with two different excitation wavelengths: DAPI channel (387/11 nm excitation filter) for the naphthalimide (mannolipid) and Cy5 channel (650/13 nm excitation filter) for Alexa 647 (ConA). Microscopy images show a strong fluorescent intensity on the surface of the droplets in both channels evidencing the binding of ConA to the mannosylated droplets (Figure 5).
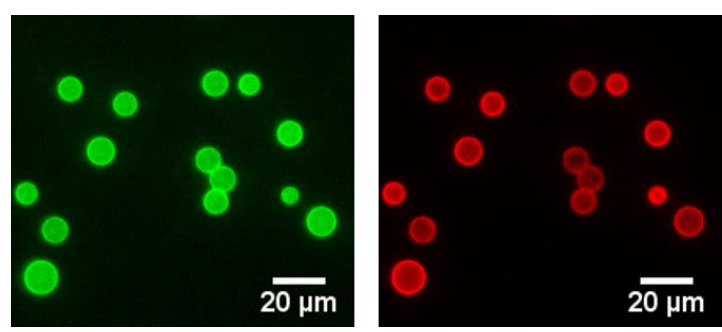

Figure 5. Pseudo-colored epifluorescence microscopy images (40X objective) of emulsion droplets coated with 1.2 surface equivalents of mannolipid and incubated with $0.24 \mu \mathrm{M}$ of ConA. Green channel (naphthalimide fluorescence) red channel (Alexa 647 fluorescence of labeled ConA).

We assessed the selectivity of the binding by performing negative control experiments with noncoated (naked) droplets and competitive binding assays with D-mannose and D-galactose on droplets coated with 0.6 eq. (i.e. $58 \mu \mathrm{M}$ ) of mannolipid (Figure 6A). Despite the presence of nonspecific binding on naked droplets, there is a specific interaction between ConA and mannosecoated droplets, since the binding can be inhibited by increasing concentrations of D-mannose, a competing ligand, but not by galactose which does not bind to ConA. A control binding experiment with Alexa 647 labeled peanut agglutinin (PNA) a galactose lectin was also performed at two different surface coating concentration, 1.2 and 6 surface equivalents. On the contrary to ConA, no binding is observed with PNA apart from a low non-specific binding that do not vary with the surface coating (Figure S4). 

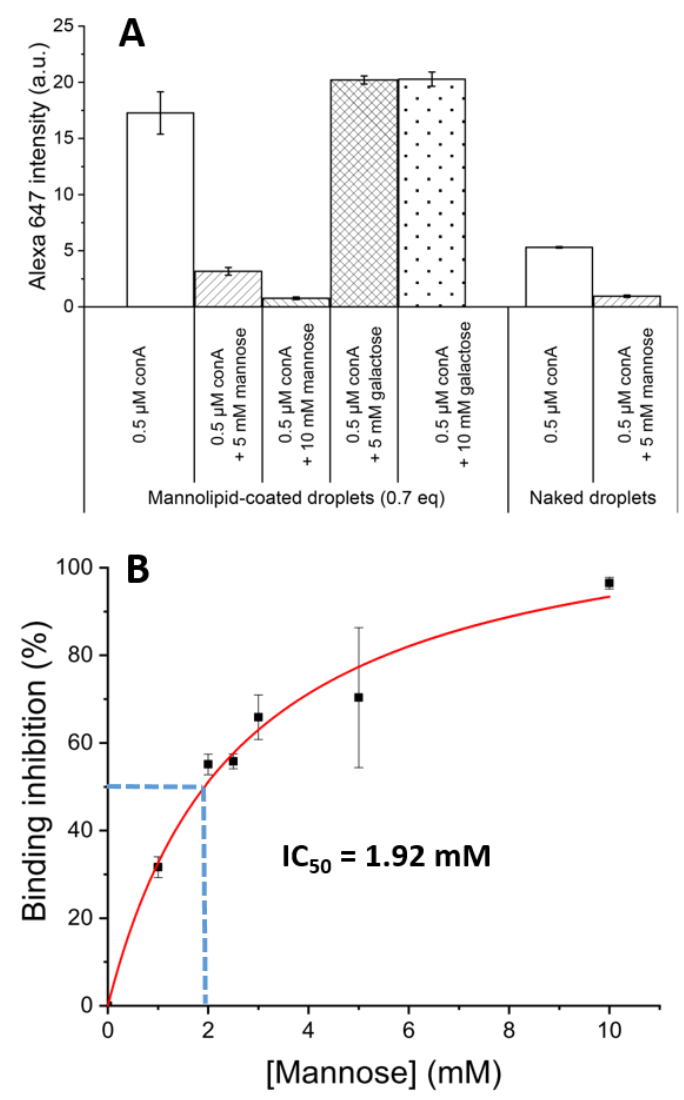

Figure 6. (A) Binding selectivity: Average intensity measured for droplets coated with 0.6 eq. of mannolipid incubated with $0.48 \mu \mathrm{M}$ of ConA and in the presence of free sugars. Control with naked droplets. (B) Competitive binding assay: Titration of ConA labeled with Alexa $647(0.48 \mu \mathrm{M})$ with mannose in the presence of droplets functionalized with 0.6 surface equivalent of mannolipid 1 (i.e. $58 \mu \mathrm{M}$ of mannolipid). Average of two independent experiments.

To measure the affinity of the mannose-coated particles, we have performed a competitive binding assay in the presence of free $\alpha-D$-mannose, which was able to completely inhibit the interaction with an $\mathrm{IC}_{50}$ of $1.92 \mathrm{mM}$ (Figure 6B). Nonlinear curve fitting yielded an apparent dissociation constant of $2.3 \mathrm{mM}$. If we consider simple model where the two ligand compete at the same binding site, we can calculate the $\mathrm{K}_{d}$ between the mannose-coated droplets and ConA. ${ }^{47}$ Considering the reported $\mathrm{K}_{d}$ of $360 \mu \mathrm{M}$ for mannose, ${ }^{37}$ we find a $\mathrm{K}_{d}$ of $9 \mu \mathrm{M}$ for the droplets coated with 0.6 surface equivalent (see SI for curve fitting and equations, Table S2).

In liposomes, the deformable surface can hinder the recognition and requires a careful design of the ligand. It has been shown in vitro and in cellulo that the recognition of glycosylated liposomes by lectins is highly dependent on the state of phase and composition of the lipid bilayers and that the ligands have to be distanced from the surface. ${ }^{48-50}$ In our design, the targeting moiety is readily exposed at the surface of the droplets without any spacing linker and still enables efficient recognition. This is in line with earlier findings that the stiffer surface of $\mathrm{O} / \mathrm{W}$ droplets facilitates 
target recognition compared to liposomes where the ligand needs to be distanced from the surface..$^{51,52}$

Overall, we thus show that $\mathrm{O} / \mathrm{W}$ emulsions are highly convenient platforms to build-up targeted particles in well-defined size and we confirm the initial rationale that the organization of monovalent sugars onto a liquid interface yields high affinity and specific lectin ligands with an affinity between ConA and the mannose-coated droplets 40 times higher than that of free mannose.

Phagocytosis of mannolipid-coated droplets. The phagocytic uptake of mannolipid-coated $8 \mu \mathrm{m}$ diameter oil droplets at two surface densities ( 0.1 and 1 surface equivalent) was assessed and compared to the uptake of uncoated droplets, using a method described previously. ${ }^{53}$ As can be seen on Figure 7A, mannolipid-coated droplets were internalized, however, fluorescence from the mannolipid can be seen in all macrophages regardless of the internalization of droplets. An average of 1.3 saturated droplets/cell were internalized, a number similar to the uptake of saturated IgGcoated $8 \mu \mathrm{m}$ diameter droplets by the same cells. ${ }^{53}$ Unsaturated droplets were less internalized, with a phagocytic index of 0.3 droplet/cell, and the uptake of uncoated droplets was significantly lower than both coated droplet assays, under 0.05 droplet/cell, as summarized on Figure 8A. On live imaging, mannolipids formed clusters at the contact site with cells, as seen on the images and plots presented in Figure 7B-D (see also supplementary movie). Live phagocytic assay presenting coated droplets to macrophages showed that the mannolipid is internalized from the solution, without any direct contact between the cell and the droplets required for the transfer. The same phenomenon occurred when soluble mannolipid was injected in the culture medium. In both cases, the internalization of mannolipid takes less than 2 minutes, as seen on SI Figure S5. 


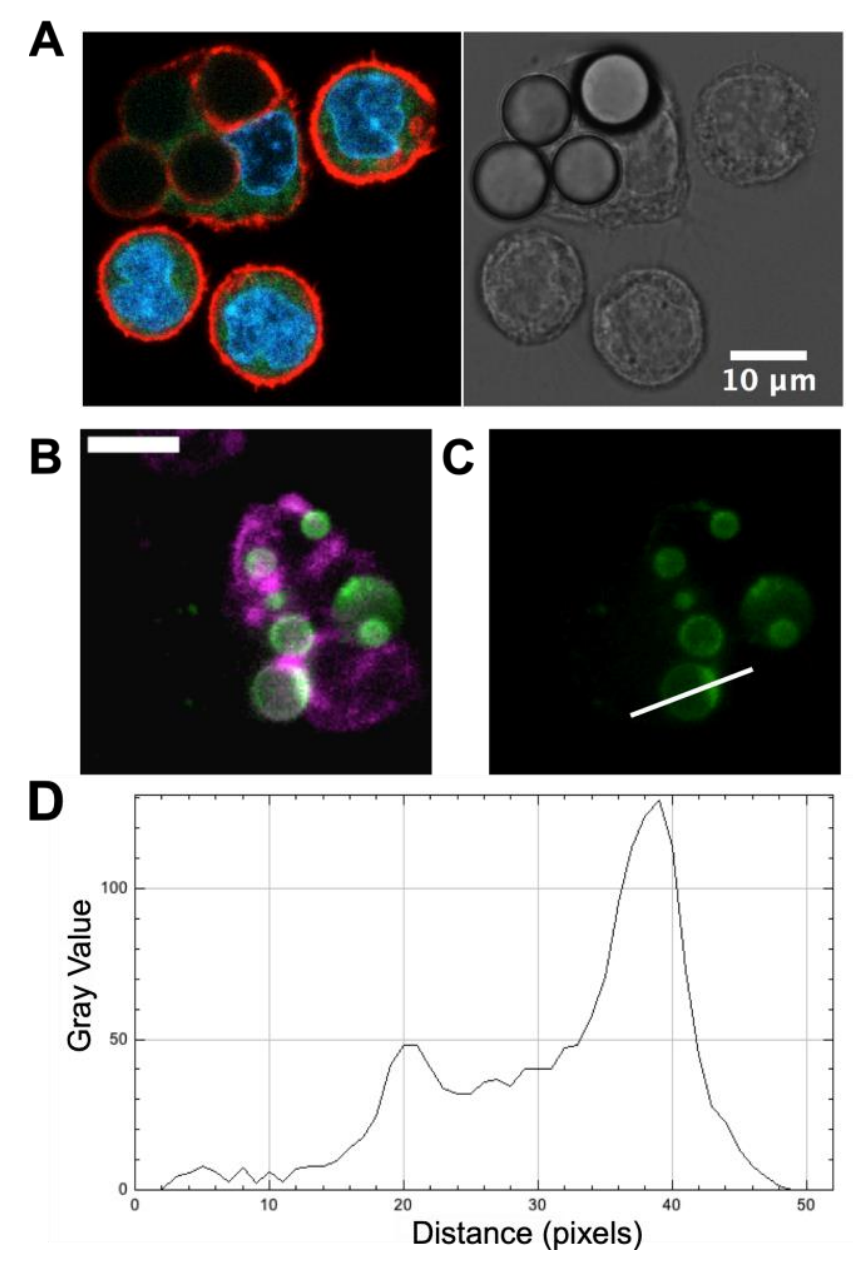

Figure 7. (A) Confocal image of macrophages presented with mannolipid-coated oil droplets (1 surface eq.). F-actin in red, mannolipid in green, nucleus in blue. Mannolipid is present in equal quantity inside all macrophages, independently of the uptake of droplets. (B-C) Live confocal image of mannolipid-coated droplets (0.1 eq, in green) in contact with macrophages (SiRactin $100 \mathrm{nM}$ in magenta). (D) Plot profile of intensity along the white line, respectively, from the cell contact toward the exterior. Clusters are formed at cell contact.

To assess whether soluble mannolipid can activate the macrophages and indirectly increase the phagocytic uptake of droplets, cells were exposed to soluble mannolipid (72 $\mu \mathrm{M})$ for 10 minutes. They were then rinsed and presented with uncoated droplets, and their uptake compared with unstimulated cells presented with coated droplets. Cells exposed to soluble mannolipid exhibited similar low levels of uptake as non-stimulated cells towards uncoated droplets (around 0.01 droplet per cell, Figure 8B), demonstrating that the presence of mannolipids on the surface of the droplets is required for internalizing the targets. 

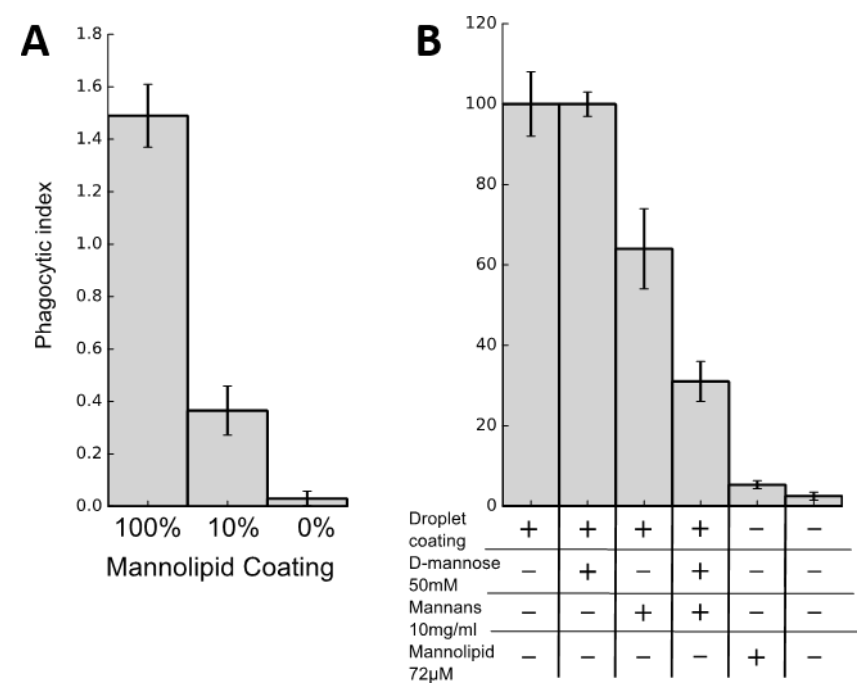

Figure 8. (A) Phagocytic index for $8 \mu \mathrm{m}$ diameter mannolipid-coated droplets (1 eq, 0.1 eq and uncoated) internalized by RAW264.7 macrophages for $45 \mathrm{~min}$ (B) Relative phagocytic index for coated (1 eq) and uncoated droplets by macrophages exposed to soluble D-mannose, mannans or mannolipid

Previous experiments showed that soluble D-mannose and mannans can impede the phagocytosis of zymosan particles by saturating cell receptors. ${ }^{12}$ We performed the same kind of experiments with mannolipid-coated droplets. While $50 \mathrm{mM}$ of D-Mannose had no discernable effect on the uptake of droplets, the addition of $10 \mathrm{mg} / \mathrm{ml}$ of yeast mannans efficiently reduced the uptake of mannolipid-coated droplets by $35 \%$. The lack of inhibition by monovalent D-mannose can easily be explained by the low affinity between CLRs and free mannose $\left(K_{d}\right.$ of $2.3 \mathrm{mM}$ reported for DCSIGN), ${ }^{54}$ but interestingly, D-mannose and mannans showed enhanced inhibition when presented together, reducing the phagocytic index by $70 \%$.

Our results show that the surface functionalization with mannose units specifically triggers the phagocytosis of micrometric emulsion droplets in a receptor-dependent process. In earlier studies aimed at studying the involvement of mannose receptors in phagocytosis, the activation of the Mannose Receptor and DC-SIGN was usually performed with yeasts ${ }^{11,55}$, bacteria ${ }^{10,56}$ or zymosan particles $^{12}$. The downside of such experiments is that other proteins at the surface of the particles can activate alternative phagocytic pathways simultaneously while our results show that uncoated $\mathrm{O} / \mathrm{W}$ emulsion droplets display very little unspecific internalization.

\section{CONCLUSION}

We have developed a supramolecular platform composed of a fluorescent mannolipid adsorbed on emulsion droplets that can be used to form targeted lipid particles able to interact with lectin with high affinity and specificity. 
The functionalized microparticles were used to activate and study the mannose receptor-mediated phagocytosis in macrophages. The phagocytosis of the microparticles was specific and receptordependent as shown by inhibition by mannans and by the negligible unspecific internalization. Compared to an alternative approach using targeted emulsions packed with lipophilic dyes, ${ }^{57}$ our work underlines the importance of developing fluorescent surface ligands: the fluorescent mannolipid developed herein allows a facile characterization of the surface functionalization by fluorescence microscopy, and also to visualize the fate of the ligands and in particular their clustering at the interface during cellular adhesion.

The mannosylated emulsion droplets developed herein bring supporting evidence of the ability of glycoreceptors to trigger phagocytosis independently of other known phagocytic receptors. Future developments are needed to isolate and identify specific receptors involved in the process. In particular, the inhibition by mannans the synergistic effect observed in combination with mannose calls for continued investigations. This specific internalization pathway can be used for the targeting and delivery of lipophilic dyes towards phagocytic cells of the immune system. Other fluorescent ligands with different sugars may also be envisioned to target additional receptors. While micrometric particles are needed to study phagocytosis, this work also opens the possibility to develop nanometric targeted fluorescent emulsions for more diverse targeting and bioimaging applications.

\section{DECLARATIONS}

\section{Supplementary informations.}

- Materials and methods

- Synthetic methods

- Additional figures and nonlinear curve fitting

- Movie of a phagocytic event

Competing interests. No financial competing interests are to be declared.

Funding. This work has received support from the administrative and technological staff of "Institut Pierre-Gilles de Gennes" (Laboratoire d'excellence : ANR-10-LABX-31, "Investissements d'avenir" : ANR-10-IDEX-0001-02 PSL and Equipement d'excellence: ANR-10-EQPX-34). J. F. and L. M. acknowledge funding from the Agence Nationale de la Recherche (ANR Jeune Chercheur 
PHAGODROP). PM, JF, JMM acknowledge funding from the PSL - Chimie doctoral program. LP acknowledge funding from Sorbonne Université IPV doctoral program.

Authors' contributions. JF, LM, BD and JMM designed the experiments; PM, BD, LM, LP and LC performed the experiments. BD, LM, JF and JMM analyzed the data and redacted the manuscript. All authors have read and approved the final manuscript.

Acknowledgements. We thank the staff of the cleanroom of the Institut Pierre-Gilles de Gennes for their help and support all throughout the project.

\section{REFERENCES}

(1) Flannagan, R. S.; Jaumouillé, V.; Grinstein, S. The Cell Biology of Phagocytosis. Annu. Rev. Pathol. Mech. Dis. 2012, 7, 61-98.

(2) Flannagan, R. S.; Harrison, R. E.; Yip, C. M.; Jaqaman, K.; Grinstein, S. Dynamic Macrophage "Probing" Is Required for the Efficient Capture of Phagocytic Targets. J. Cell Biol. 2010, 191, 1205-1218.

(3) Ben M'Barek, K.; Molino, D.; Quignard, S.; Plamont, M. A.; Chen, Y.; Chavrier, P.; Fattaccioli, J. Phagocytosis of Immunoglobulin-Coated Emulsion Droplets. Biomaterials 2015, 51, 270277.

(4) Headley, M. B.; Roberts, E. W.; Kern, N.; Steinbach, A. M.; Williamson, A. P.; Vale, R. D.; Morrissey, M. A. Chimeric Antigen Receptors That Trigger Phagocytosis. Elife 2018, 7, e36688.

(5) Swanson, J. A. Shaping Cups into Phagosomes and Macropinosomes. Nat. Rev. Mol. Cell Biol. 2008, 9, 639-649.

(6) Swanson, J. A. Cell Biology: The Extraordinary Phagosome. Nature 2002, 418, $286-287$.

(7) McGreal, E. P.; Miller, J. L.; Gordon, S. Ligand Recognition by Antigen-Presenting Cell CType Lectin Receptors. Curr. Opin. Immunol. 2005, 17, 18-24.

(8) Martinez-Pomares, L. The Mannose Receptor. J. Leukoc. Biol. 2012, 92, 1177-1186.

(9) Hardison, S. E.; Brown, G. D. C-Type Lectin Receptors Orchestrate Antifungal Immunity. Nat. Immunol. 2012, 13, 817-822. 
(10) Tailleux, L.; Pham-Thi, N.; Bergeron-Lafaurie, A.; Herrmann, J.-L.; Charles, P.; Schwartz, O.; Scheinmann, P.; Lagrange, P. H.; Blic, J. de; Tazi, A.; Gicquel, B.; Neyrolles, O. DC-SIGN Induction in Alveolar Macrophages Defines Privileged Target Host Cells for Mycobacteria in Patients with Tuberculosis. PLoS Med. 2005, 2, e381.

(11) Cambi, A.; Netea, M. G.; Mora-Montes, H. M.; Gow, N. A. R.; Hato, S. V.; Lowman, D. W.; Kullberg, B. J.; Torensma, R.; Williams, D. L.; Figdor, C. G. Dendritic Cell Interaction with Candida Albicans Critically Depends on N-Linked Mannan. J. Biol. Chem. 2008, 283, 2059020599.

(12) Sung, S. S. J.; Nelson, R. S.; Silverstein, S. C. Yeast Mannans Inhibit Binding and Phagocytosis of Zymosan by Mouse Peritoneal Macrophages. J. Cell Biol. 1983, 96, 160166.

(13) Serrano-Gómez, D.; Domínguez-Soto, A.; Ancochea, J.; Jimenez-Heffernan, J. A.; Leal, J. A.; Corbí, A. L. Dendritic Cell-Specific Intercellular Adhesion Molecule 3-Grabbing Nonintegrin Mediates Binding and Internalization of Aspergillus Fumigatus Conidia by Dendritic Cells and Macrophages. J. Immunol. 2004, 173, 5635 - 5643.

(14) Ezekowitz RA, Sastry K, Bailly P, W. A. Molecular Characterization of the Human Macrophage Mannose Receptor: Demonstration of Multiple Carbohydrate Recognition-like Domains and Phagocytosis of Yeasts in Cos-1 Cells. J. Exp. Med. 1990, 172, 1785-1794.

(15) Le Cabec, V.; Emorine, L. J.; Toesca, I.; Cougoule, C.; Maridonneau-Parini, I. The Human Macrophage Mannose Receptor Is Not a Professional Phagocytic Receptor. J. Leukoc. Biol. 2005, 77, 934-943.

(16) Yu, H.; Xiao, Y.; Jin, L. A Lysosome-Targetable and Two-Photon Fluorescent Probe for Monitoring Endogenous and Exogenous Nitric Oxide in Living Cells. J. Am. Chem. Soc. 2012, $134,17486-17489$.

(17) Fernández, A.; Vendrell, M. Smart Fluorescent Probes for Imaging Macrophage Activity. Chem. Soc. Rev. 2016, 45, 1182-1196.

(18) Vázquez-Romero, A.; Kielland, N.; Arévalo, M. J.; Preciado, S.; Mellanby, R. J.; Feng, Y.; Lavilla, R.; Vendrell, M. Multicomponent Reactions for de Novo Synthesis of BODIPY Probes: In Vivo Imaging of Phagocytic Macrophages. J. Am. Chem. Soc. 2013, 135, 16018-16021.

(19) Kenmoku, S.; Urano, Y.; Kojima, H.; Nagano, T. Development of a Highly Specific Rhodamine-Based Fluorescence Probe for Hypochlorous Acid and Its Application to RealTime Imaging of Phagocytosis. J. Am. Chem. Soc. 2007, 129, 7313-7318. 
(20) Wolfbeis, O. S. An Overview of Nanoparticles Commonly Used in Fluorescent Bioimaging. Chem. Soc. Rev. 2015, 44, 4743-4768.

(21) Howes, P. D.; Chandrawati, R.; Stevens, M. M. Colloidal Nanoparticles as Advanced Biological Sensors. Science 2014, 346, 1247390-1247390.

(22) Faraji, A. H.; Wipf, P. Nanoparticles in Cellular Drug Delivery. Bioorganic Med. Chem. 2009, 17, 2950-2962.

(23) Hu, J.; Wei, P.; Seeberger, P. H.; Yin, J. Mannose-Functionalized Nanoscaffolds for Targeted Delivery in Biomedical Applications. Chem. As. J. 2018, 13, 3448-3459.

(24) Mauceri, A.; Borocci, S.; Galantini, L.; Giansanti, L.; Mancini, G.; Martino, A.; Salvati Manni, L.; Sperduto, C. Recognition of Concanavalin a by Cationic Glucosylated Liposomes. Langmuir 2014, 30, 11301-11306.

(25) Perche, F.; Benvegnu, T.; Berchel, M.; Lebegue, L.; Pichon, C.; Jaffrès, P. A.; Midoux, P. Enhancement of Dendritic Cells Transfection in Vivo and of Vaccination against B16F10 Melanoma with Mannosylated Histidylated Lipopolyplexes Loaded with Tumor Antigen Messenger RNA. Nanomedicine Nanotechnology, Biol. Med. 2011, 7, 445-453.

(26) Espuelas, S.; Thumann, C.; Heurtault, B.; Schuber, F.; Frisch, B. Influence of Ligand Valency on the Targeting of Immature Human Dendritic Cells by Mannosylated Liposomes. Bioconjug. Chem. 2008, 19, 2385-2393.

(27) Tamilvanan, S. Oil-in-Water Lipid Emulsions: Implications for Parenteral and Ocular Delivering Systems. Prog. Lipid Res. 2004, 43, 489-533.

(28) Leal-Calderon, F.; Schmitt, V.; Bibette, J. Emulsion Science: Basic Principles, 2nd ed.; Springer, 2007.

(29) Bourouina, N.; Husson, J.; Hivroz, C.; Henry, N. Biomimetic Droplets for Artificial Engagement of Living Cell Surface Receptors: The Specific Case of the T-Cell. Langmuir 2012, 28, 6106-6113.

(30) Fattaccioli, J.; Baudry, J.; Henry, N.; Brochard-Wyart, F.; Bibette, J. Specific Wetting Probed with Biomimetic Emulsion Droplets. Soft Matter 2008, 4, 2434-2440.

(31) Pontani, L.-L.; Jorjadze, I.; Viasnoff, V.; Brujic, J. Biomimetic Emulsions Reveal the Effect of Mechanical Forces on Cell-Cell Adhesion. Proc. Natl. Acad. Sci. 2012, 109, 9839-9844.

(32) Bourouina, N.; Husson, J.; Waharte, F.; Pansu, R. B.; Henry, N. Formation of Specific Receptor-Ligand Bonds between Liquid Interfaces. Soft Matter 2011, 7, 9130. 
(33) Zhang, Q.; Scigliano, A.; Biver, T.; Pucci, A.; Swager, T. M. Interfacial Bacsioconjugation on Emulsion Droplet for Biosensors. Bioorg. Med. Chem. 2018, 26, 5307-5313.

(34) Cecioni, S.; Imberty, A.; Vidal, S. Glycomimetics versus Multivalent Glycoconjugates for the Design of High Affinity Lectin Ligands. Chem. Rev. 2015, 115, 525-561.

(35) He, X. P.; Zang, Y.; James, T. D.; Li, J.; Chen, G. R.; Xie, J. Fluorescent Glycoprobes: A Sweet Addition for Improved Sensing. Chem. Commun. 2017, 53, 82-90.

(36) He, X.; Tian, H. Lightening Up Membrane Receptors with Fluorescent Molecular Probes and Supramolecular Materials. Chem 2018, 4, 246-268.

(37) Swaminathan, C. P.; Surolia, N.; Surolia, A. Role of Water in the Specific Binding of Mannose and Mannooligosaccharides to Concanavalin A. J. Am. Chem. Soc. 1998, 120, 5153-5159.

(38) Mangold, S. L.; Prost, L. R.; Kiessling, L. L. Quinoxalinone Inhibitors of the Lectin DC-SIGN. Chem. Sci. 2012, 3, 772-777.

(39) Cattiaux, L.; Porkolab, V.; Fieschi, F.; Mallet, J. M. New Branched Amino Acids for High Affinity Dendrimeric DC-SIGN Ligands. Bioorganic Med. Chem. 2018, 26, 1006-1015.

(40) Saha, S.; Samanta, A. Influence of the Structure of the Amino Group and Polarity of the Medium on the Photophysical Behavior of 4-Amino-1,8-Naphthalimide Derivatives. J. Phys. Chem. A 2002, 106, 4763-4771.

(41) Liu, X.; Qiao, Q.; Tian, W.; Liu, W.; Chen, J.; Lang, M. J.; Xu, Z. Aziridinyl Fluorophores Demonstrate Bright Fluorescence and Superior Photostability by Effectively Inhibiting Twisted Intramolecular Charge Transfer. J. Am. Chem. Soc. 2016, 138, 6960-6963.

(42) Pinon, L.; Montel, L.; Mesdjian, O.; Bernard, M.; Michel, A.; Ménager, C.; Fattaccioli, J. Kinetically Enhanced Fabrication of Homogeneous Biomimetic and Functional Emulsion Droplets. Langmuir 2018, 34, 15319-15326.

(43) Mason, T.; Bibette, J. Emulsification in Viscoelastic Media. Phys. Rev. Lett. 1996, 77, 34813484.

(44) Sekhar, K. P. C.; Bangal, P. R.; Nayak, R. R. A Systematic Surface Studies on the Glycolipids to Understand the Surface Adsorption Behavior. Colloids Surfaces $A$ Physicochem. Eng. Asp. 2019, 563, 226-236.

(45) Reisch, A.; Klymchenko, A. S. Fluorescent Polymer Nanoparticles Based on Dyes: Seeking Brighter Tools for Bioimaging. Small 2016, 12, 1968-1992.

(46) Chervenak, M. C.; Toone, E. J. Calorimetric Analysis of the Binding of Lectins with 
Overlapping Carbohydrate-Binding Ligand Specificities. Biochemistry 1995, 34, 5685-5695.

(47) Hulme, E. C.; Trevethick, M. A. Ligand Binding Assays at Equilibrium: Validation and Interpretation. Br. J. Pharmacol. 2010, 161, 1219-1237.

(48) Engel, A.; Chatterjee, S. K.; Al-Arifi, A.; Nuhn, P. Influence of Spacer Length on the Agglutination of Glycolipid-Incorporated Liposomes by ConA as Model Membrane. J. Pharm. Sci. 2003, 92, 2229-2235.

(49) Jeong, H. S.; Na, K. S.; Hwang, H.; Oh, P. S.; Kim, D. H.; Lim, S. T.; Sohn, M. H.; Jeong, H. J. Effect of Space Length of Mannose Ligand on Uptake of Mannosylated Liposome in RAW 264.7 Cells: In Vitro and in Vivo Studies. J. Biomed. Mater. Res. - Part A 2014, 102, 45454553.

(50) Villalva, D. G.; Giansanti, L.; Mauceri, A.; Ceccacci, F.; Mancini, G. Influence of the State of Phase of Lipid Bilayer on the Exposure of Glucose Residues on the Surface of Liposomes. Colloids Surfaces B Biointerfaces 2017, 159, 557-563.

(51) Yeeprae, W.; Kawakami, S.; Yamashita, F.; Hashida, M. Effect of Mannose Density on Mannose Receptor-Mediated Cellular Uptake of Mannosylated O/W Emulsions by Macrophages. J. Control. Release 2006, 114, 193-201.

(52) Zhang, Q.; Savagatrup, S.; Kaplonek, P.; Seeberger, P. H.; Swager, T. M. Janus Emulsions for the Detection of Bacteria. ACS Cent. Sci. 2017, 3, 309-313.

(53) Montel, L.; Pinon, L.; Fattaccioli, J. A Multiparametric and High-Throughput Assay to Quantify the Influence of Target Size on Phagocytosis. Biophys. J. 2019, 117 (3), 408-419.

(54) Feinberg, H.; Castelli, R.; Drickamer, K.; Seeberger, P. H.; Weis, W. I. Multiple Modes of Binding Enhance the Affinity of DC-SIGN for High Mannose N-Linked Glycans Found on Viral Glycoproteins. J. Biol. Chem. 2007, 282, 4202-4209.

(55) Ezekowitz, R. A. B.; Sastry, K.; Bailly, P.; Warner, A. Molecular Characterization of the Human Macrophage Mannose Receptor: Demonstration of Multiple Carbohydrate Recognition-like Domains and Phagocytosis of Yeasts in Cos-1 Cells. J. Exp. Med. 1990, 172, 1785-1794.

(56) Ackerman, M. E.; Moldt, B.; Wyatt, R. T.; Dugast, A.-S.; McAndrew, E.; Tsoukas, S.; Jost, S.; Berger, C. T.; Sciaranghella, G.; Liu, Q.; Irvine, D. J.; Burton, D. R.; Alter, G. A Robust, HighThroughput Assay to Determine the Phagocytic Activity of Clinical Antibody Samples. J. Immunol. Methods 2011, 366, 8-19.

Klymchenko, A. S.; Roger, E.; Anton, N.; Anton, H.; Shulov, I.; Vermot, J.; Mely, Y.; 
Vandamme, T. F. Highly Lipophilic Fluorescent Dyes in Nano-Emulsions: Towards Bright Non-Leaking Nano-Droplets. RSC Adv. 2012, 2, 11876. 
TOC graphic

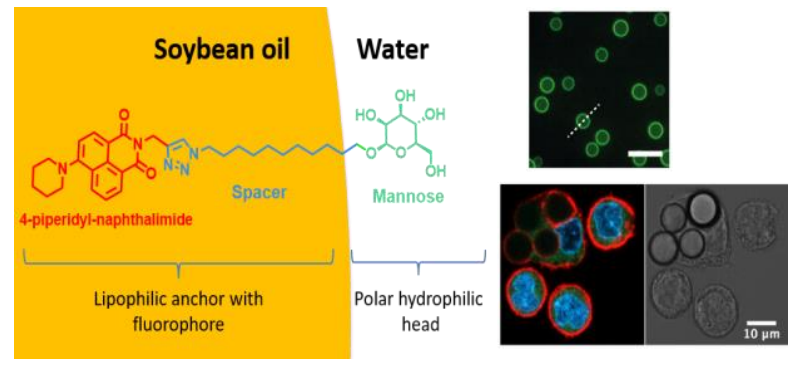

